\title{
Efficient Quantum Gates for Individual Nuclear Spin Qubits by Indirect Control
}

\author{
Swathi S. Hegde, Jingfu Zhang, and Dieter Suter \\ Fakultät Physik, Technische Universität Dortmund, D-44221 Dortmund, Germany
}

\begin{abstract}
Hybrid quantum registers, such as electron-nuclear spin systems, have emerged as promising hardware for implementing quantum information and computing protocols in scalable systems. Nevertheless, the coherent control of such systems still faces challenges. Particularly, the lower gyromagnetic ratios of the nuclear spins cause them to respond slowly to control fields, resulting in gate times that are generally longer than the coherence time of the electron. Here, we demonstrate a scheme for circumventing this problem by indirect control: we apply a small number of short pulses only to the electron and let the full system undergo free evolution under the hyperfine coupling between the pulses. Using this scheme, we realize robust quantum gates in an electron-nuclear spin system, including a Hadamard gate on the nuclear spin and a controlled-NOT gate with the nuclear spin as the target qubit. The durations of these gates are shorter than the electron coherence time, and thus additional operations to extend the system coherence time are not needed. Our demonstration serves as a proof of concept for achieving efficient coherent control of electron-nuclear spin systems, such as NV centers in diamond. Our scheme is still applicable when the nuclear spins are only weakly coupled to the electron.
\end{abstract}

Spin-based quantum registers have come up as a feasible architecture for implementing quantum computing $[1,2]$. Among them are the hybrid systems consisting of electron and nuclear spins such as Nitrogen Vacancy (NV) centers in diamond [3-13]. Specific properties of their subsystems are the distinct gyromagnetic ratios, which result, e.g. in the requirement that the frequencies of the control fields applied to electronic and nuclear spins lie in the microwave (MW) and radiofrequency (RF) regimes respectively. The fast gate operation times on the electrons (order of ns) and the long coherence times of the nuclear spins (order of ms) serve as efficient control and memory channels. However, the lower gyromagnetic ratios of the nuclear spins result in longer nuclear spin gate operation times (a few tens of $\mu \mathrm{s}$ ), which can exceed the electron coherence times $(\approx 1-25 \mu \mathrm{s})$ at room temperature, thus posing a major challenge for coherent control of electron-nuclear spin systems. Techniques like dynamical decoupling (DD) can partly alleviate this issue by extending the coherence times of the electron [14-19], but the additional DD pulses increase the control cost.

Previously, one- and two-qubit operations were demonstrated using RF pulses on the nuclear spin that had strong hyperfine coupling of $\approx 130 \mathrm{MHz}[20-22]$. Such strong couplings enhance the nuclear spin Rabi frequency allowing fast RF operations (order of ns) and hence direct control of nuclear spins was feasible [21, 23, 24]. However, scalable quantum computing requires coherent control of tens to hundreds of qubits and the control of dipolar coupled nuclear spins gets challenging with increasing distance from the electrons. To avoid these challenges, indirect control (IC) of the nuclear spins has also been incorporated [25-30]. In this approach, the control fields are applied only on the electron, combined with free evolution of the system under the hyperfine couplings. However, most of the earlier works based on IC required a large number of control operations, thereby increasing the control overhead $[28,31]$.

In this letter, we experimentally implement efficient quantum gates in an NV center in diamond at room temperature, using IC with minimal control cost of only 2-3 of short MW pulses and delays. Our approach allows variable delays and pulse parameters. As such, it differs from earlier work [31] that used many DD cycles with fixed delays. We use this approach to demonstrate quantum gates that are required for a universal set of gates: a Hadamard gate on a nuclear spin, and a controlled-NOT (CNOT) gate with control on the electron and target on the nuclear spin.

We consider a single NV center that consists of a spin-1 electron coupled to a spin- $1{ }^{14} \mathrm{~N}$ and a spin- $1 / 2{ }^{13} \mathrm{C}$ (see Supplemental Material [32]). We perform the operations on the electron and ${ }^{13} \mathrm{C}$ by focussing on a subspace of the system where the ${ }^{14} \mathrm{~N}$ is in the $m_{N}=1$ state. We then can write the secular part of the electron- ${ }^{13} \mathrm{C}$ Hamiltonian in the lab frame as $\mathcal{H} /(2 \pi)=D\left(S_{z}^{2} \otimes E_{2}\right)-\left(\nu_{e}-\right.$ $\left.A_{N}\right)\left(S_{z} \otimes E_{2}\right)-\nu_{C}\left(E_{3} \otimes I_{z}\right)+A_{z z}\left(S_{z} \otimes I_{z}\right)+A_{z x}\left(S_{z} \otimes I_{x}\right)$, where $S_{z}$ and $I_{z / x}$ are the spin operators for electron and ${ }^{13} \mathrm{C}=$ respectively, $E_{n}$ is an $n \times n$ identity matrix, $D=2.87 \mathrm{GHz}$ is the zero field splitting, $\nu_{e}=-414 \mathrm{MHz}$ and $\nu_{C}=0.158 \mathrm{MHz}$ are the Larmor frequencies of the electron and ${ }^{13} \mathrm{C}$ in a $14.8 \mathrm{mT}$ field, $A_{N}=-2.16 \mathrm{MHz}$ is the hyperfine coupling with ${ }^{14} \mathrm{~N}$ and $A_{z z}=-0.152$ $\mathrm{MHz}$ and $A_{z x}=0.110 \mathrm{MHz}$ are the hyperfine couplings with ${ }^{13} \mathrm{C}$. The eigenstates of $\mathcal{H}$ are $|0 \uparrow\rangle, \mid 0 \downarrow$ \rangle$,\left|-1 \varphi_{-}\right\rangle,\left|-1 \psi_{-}\right\rangle,\left|1 \varphi_{+}\right\rangle,\left|1 \psi_{+}\right\rangle$, where $\{|0\rangle,| \pm 1\rangle\}$ are the eigenstates of $S_{z}$, and

$$
\begin{aligned}
& \left|\varphi_{ \pm}\right\rangle=\cos \left(\kappa_{ \pm} / 2\right)|\uparrow\rangle+\sin \left(\kappa_{ \pm} / 2\right)|\downarrow\rangle \\
& \left|\psi_{ \pm}\right\rangle=-\sin \left(\kappa_{ \pm} / 2\right)|\uparrow\rangle+\cos \left(\kappa_{ \pm} / 2\right)|\downarrow\rangle .
\end{aligned}
$$

Here $\{|\uparrow\rangle,|\downarrow\rangle\}$ are the eigenstates of $I_{z}$, and $\kappa_{ \pm}=$ $\arctan \left[A_{z x} /\left(A_{z z} \mp \nu_{C}\right)\right]$ is the angle between the quantization axis of the ${ }^{13} \mathrm{C}$ and the NV axis.

We implement the quantum gates $U_{T}$ in the $m_{S}=$ $\{0,-1\}$ and $m_{N}=1$ manifold and refer to it as the system subspace. This choice of subspace is realized by 


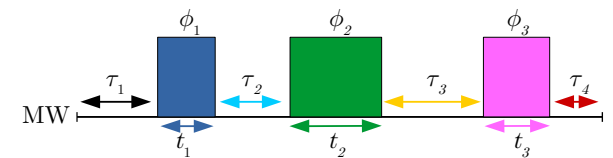

FIG. 1: MW pulse sequence to realize $U_{T}$ by IC, at a fixed $\omega_{1}$. The delays $\tau_{i}$, MW pulse durations $t_{i}$ and phases $\phi_{i}$ are the free variables to be optimized.

using MW pulses with a Rabi frequency of $\approx 0.5 \mathrm{MHz}$ $\left(\ll A_{N}\right)$, which covers all electron spin resonance (ESR) transitions in the system subspace but leaves states untouched where the ${ }^{14} \mathrm{~N}$ is in a different state. For the system subspace, the Hamiltonian is $\mathcal{H}_{s} /(2 \pi)=$ $|0\rangle\left\langle 0\left|\otimes \mathcal{H}_{0}+\right|-1\right\rangle\langle-1| \otimes \mathcal{H}_{-1}$, where $\mathcal{H}_{0}=-\nu_{C} I_{z}$ and $\mathcal{H}_{-1}=-\left(\nu_{C}+A_{z z}\right) I_{z}-A_{z x} I_{x}$ are ${ }^{13} \mathrm{C}$ spin Hamiltonians when the electron is in $|0\rangle$ or $|-1\rangle$, respectively.

We implement two examples of $U_{T}$ :

$$
\begin{aligned}
U_{H} & =E_{2} \otimes\left[\begin{array}{cc}
1 & 1 \\
1 & -1
\end{array}\right] / \sqrt{2} \\
U_{C N O T} & =|0\rangle\left\langle 0\left|\otimes E_{2}+\right|-1\right\rangle\langle-1| \otimes e^{-i \pi I_{x}} .
\end{aligned}
$$

The first is a Hadamard gate while the second is a CNOT gate, both targeting ${ }^{13} \mathrm{C}$, in a basis defined in Ref. [33]. To check the implementation of $U_{T}$, we initialize the system into a pure state, apply $U_{T}$ and then perform a partial tomography of the final state by recording free precession signals (FIDs).

For practical applications, it is useful to allow additional degrees of freedom, such as variable pulse rotation angles and finite pulse durations. These degrees of freedom allow us to compensate experimental errors via numerical optimization of the pulse sequence parameters. As shown in Fig. 1, we consider a pulse sequence consisting of delays $\tau_{i}$ and MW pulses with durations $t_{i}$ and phases $\phi_{i}$ where $i=1 \cdots n, n$ is the number of pulses. We fix the frequency of the pulses to be resonant with the ESR transition $0 \leftrightarrow-1$ and the Rabi frequency $\omega_{1} / 2 \pi$ to $0.5 \mathrm{MHz}$. During $\tau_{i}$, the system freely evolves under $\mathcal{H}_{s}$ such that $U_{i}^{f}=e^{-i \mathcal{H}_{s} \tau_{i}}$. The control Hamiltonians during the MW pulse segments are $\mathcal{H}_{i}^{M W}=\omega_{1}\left[\cos \phi_{i}\left(s_{x} \otimes E_{2}\right)+\sin \phi_{i}\left(s_{y} \otimes E_{2}\right)\right]+\mathcal{H}_{s}$, where $s_{x / y}$ denote the spin-1/2 operators for the electron, and the corresponding operators are $U_{i}^{M W}=e^{-i \mathcal{H}_{i}^{M W} t_{i}}$. The total propagator $U$ is the time ordered product of $U_{i}^{f}$ and $U_{i}^{M W}$. The overlap between $U$ and $U_{T}$ is defined by the fidelity $F=\left|\operatorname{Tr}\left(U^{\dagger} U_{T}\right)\right| / 4$. We maximize $F$ numerically, using a MATLAB ${ }^{\mathbb{S}}$ subroutine implementing a genetic algorithm [34]. The solution returns the pulse sequence parameters $t_{i}, \tau_{i}$ and $\phi_{i}$. The sequences were made robust against fluctuations of the MW pulse amplitude by optimizing $F$ over a range $\omega_{1} /(2 \pi)=[0.48,0.52]$ $\mathrm{MHz}$. Table I summarizes the optimized pulse parameters for $U_{H}$ and $U_{C N O T}$, and the average gate fidelities are $>96 \%$ and $>97 \%$, respectively. The resulting trajectories of the electron and ${ }^{13} \mathrm{C}$ on the Bloch-sphere is shown in the Supplemental Material [32].
TABLE I: MW pulse sequence parameters for $U_{H}$ and $U_{C N O T}$. The time durations and phases are in units of $\mu \mathrm{s}$ and radians, respectively.

\begin{tabular}{|c|c|c|c|c|c|c|c|c|c|c|}
\hline & $\tau_{1}$ & $\tau_{2}$ & $\tau_{3}$ & $\tau_{4}$ & $t_{1}$ & $t_{2}$ & $t_{3}$ & $\phi_{1}$ & $\phi_{2}$ & $\phi_{3}$ \\
\hline$U_{H}$ & 0.74 & 0.22 & 0.43 & 0.89 & 0.23 & 1.26 & 1.50 & $3 \pi / 2$ & $3 \pi / 2$ & $\pi / 2$ \\
\hline$U_{C N O T}$ & 3.78 & 2.11 & 2.15 & 0.63 & 1.88 & 3.96 & 1.90 & 0 & $\pi / 5$ & $\pi / 2$ \\
\hline
\end{tabular}

Our experiments started with an initial laser pulse with a wavelength of $532 \mathrm{~nm}$, a duration of $5 \mu \mathrm{s}$, and a power of $\approx 0.5 \mathrm{~mW}$ which initialized the electron to $|0\rangle$ but left the ${ }^{13} \mathrm{C}$ in a mixed state. To initialize ${ }^{13} \mathrm{C}$ to $|\uparrow\rangle$, we resorted to the IC method $[32,35,36]$. Starting from $\psi_{0}=|0 \uparrow\rangle$, we implemented the circuits shown in Figs. $(2,3)$. Depending on the experiment, we either observed the electron or the ${ }^{13} \mathrm{C}$ state via FID measurements. The readout process consisted of another laser pulse with the same wavelength and $400 \mathrm{~ns}$ duration and was used to measure the population of $m_{S}=0$.

Figure 2(a) shows the pulse sequence for implementing and detecting the effect of $U_{H}$. The first $U_{H}$ generates $|0\rangle \otimes(|\uparrow\rangle+|\downarrow\rangle) / \sqrt{2}$. The ${ }^{13} \mathrm{C}$ coherence is then allowed to evolve for a variable time $t$ after which we apply another $U_{H}$ to convert one component of the coherence to population. Lastly, a clean-up operation, with MW pulse sequence $\left(90_{x}-\tau_{c}-90_{y}\right)$, where $90_{x / y}$ are pulses with rotation angle $90^{\circ}$ about the $x / y$-axis applied to the $m_{S}=0 \leftrightarrow 1$ transition with $0.5 \mathrm{MHz}$ Rabi frequency and $\tau_{c}=1 /\left(2\left|A_{z z}\right|\right)$ is the delay, represented by the dotted box transfers the population from $|0 \downarrow\rangle$ to $|1 \downarrow\rangle$. The final read-out operation thus detects only the population of $|0 \uparrow\rangle$, which depends on $t$ as $\left[1+\cos \left(2 \pi \nu_{C} t\right)\right] / 2$. In the frequency domain, this corresponds to a peak at $\nu_{C}$.

Using the pulse sequence in Fig. 2(a), we performed two
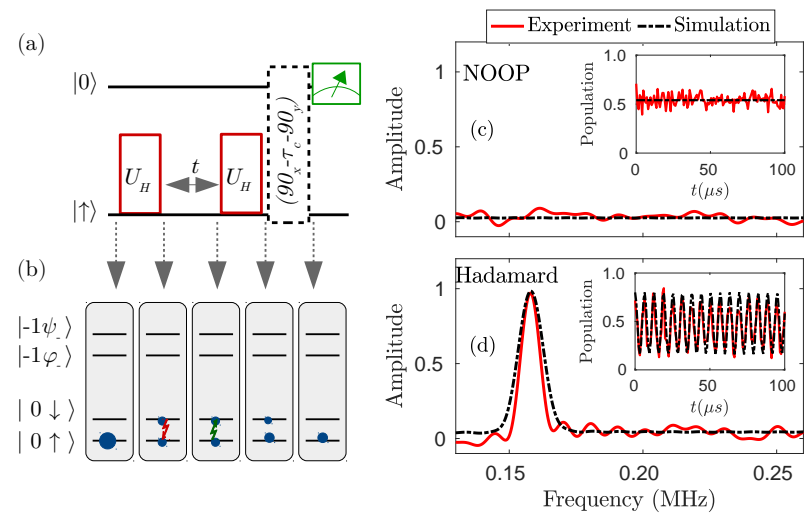

FIG. 2: (a) Quantum circuit to test $U_{H}$. The MW pulse sequence parameters for $U_{H}$ are given in Table. I. The clean-up operation is represented by the dotted box. (b) Populations (solid circles) and coherences (zig-zag arrows) at each stage of the pulse sequence in (a). (c, d) ${ }^{13} \mathrm{C}$ spin spectra obtained by the pulse sequence in (a). (c) Without the first $U_{H}$. (d) With both $U_{H}$. Inset: Final population of $|0 \uparrow\rangle$ as a function of $t$. 


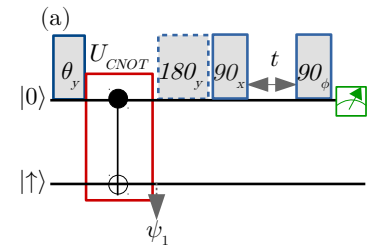

(b)

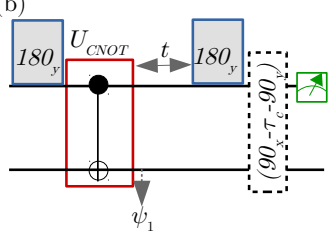

(c)

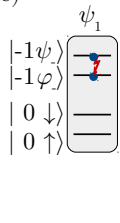

FIG. 3: Quantum circuits to test $U_{C N O T}$. The MW pulse sequence parameters for $U_{C N O T}$ indicated by red empty boxes are given in Table. I. $\theta_{x / y / \phi}$ denote operations with rotation angles $\theta$ about the $x / y / \phi$ axes that are resonant with the transition $0 \leftrightarrow-1$ and with Rabi frequencies of $8 \mathrm{MHz}$. (a) Pulse sequence to demonstrate the effect of $U_{C N O T}$ on different input states via electron spin detection. $\phi$ is the detuning phase. In the presence (absence) of the $180_{y}$ operation indicated by the dashed box, the FID measurement is used to determine the population of the $m_{S}=-1\left(m_{S}=0\right)$ after $U_{C N O T}$. (b) Pulse sequence to demonstrate the effect of $U_{C N O T}$ via ${ }^{13} \mathrm{C}$ spin detection. (c) Pictorial representation of state $\psi_{1}$.

experiments to compare the effect of $U_{H}$ : (1) without the first $U_{H}$ (i.e, no operation, also known as NOOP) and (2) with both $U_{H}$. In the case of NOOP, the system was in $\psi_{0}$ during the free evolution period. Since $\psi_{0}$ does not contain ${ }^{13} \mathrm{C}$ coherence the resulting frequency domain signal does not contain a resonance at $\nu_{C}$, as shown in Fig. 2(c). With both $U_{H}$ present, we observe in Fig. 2(d) a resonance peak at $\nu_{C}$ as expected. We numerically simulated the pulse sequence in Fig. 2(a) without and with the first $U_{H}$, and then calculated the final populations of $|0 \uparrow\rangle$ as a function of $t$. To match the theoretical signal with the experimental one, we had to scale it by a factor 0.9 for NOOP and 0.8 for $U_{H}$ (i.e, with two $\left.U_{H}\right)$, and estimated the infidelity of the experimental $U_{H}$ as $\approx 10 \%$.

The schemes to demonstrate $U_{C N O T}$ are shown in Fig. 3. Using the pulse sequence in Fig. 3(a), we demonstrated the effect of $U_{C N O T}$ in $m_{S}=-1$ by measuring electron spin spectra. Choosing for the flip-angle $\theta$ of the initial $\theta_{y}$ operation $[37,38]$ a value of $\pi$, we exchanged the populations of the $|0 \uparrow\rangle \leftrightarrow|-1 \uparrow\rangle \approx|-1\rangle \otimes\left(\left|\phi_{-}\right\rangle-\left|\psi_{-}\right\rangle\right) / \sqrt{2}$ according to Eq. (1). The subsequent $U_{C N O T}$ transformed $|-1 \uparrow\rangle$ to $-i|-1 \downarrow\rangle \approx-i|-1\rangle \otimes\left(\left|\phi_{-}\right\rangle+\left|\psi_{-}\right\rangle\right) / \sqrt{2}$, since by definition of Eq. (2), $U_{C N O T}$ flips the ${ }^{13} \mathrm{C}$ state when the electron is in $|-1\rangle$. To measure the state after $U_{C N O T}$, we transferred the population of $|-1 \downarrow\rangle$ to $|0 \downarrow\rangle$ using a hard $180_{y}$ operation. The readout process, which measures the population of $m_{S}=0$, can then be used to determine the population left in $|-1 \downarrow\rangle$ by $U_{C N O T}$. The sequence $\left(90_{x}-t-90_{\phi}\right)$ in Fig. 3(a) implements the electron spin FID measurement, where the $90_{x}$ pulse creates electron coherence and the $90_{\phi}$ pulse converts one component of the evolved coherence to population $[6,36]$. Here we incremented the phase $\phi(t)=-2 \pi \nu_{d} t$ linearly with $t$, using a detuning frequency $\nu_{d}$ of $3 \mathrm{MHz}$. We then measured the population of $m_{S}=0$ with the readout laser pulse as a function of $t$ and its Fourier transform (a)

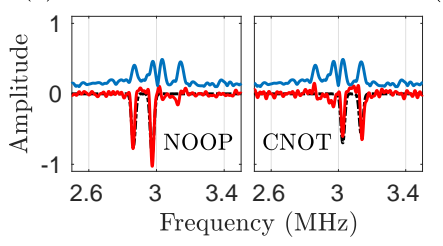

(b)

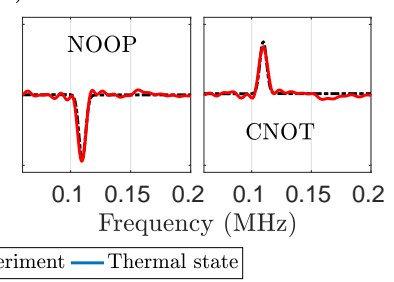

FIG. 4: (a) Electron spin spectra for the pulse sequence corresponding to Fig. 3(a) without and with $U_{C N O T}$ where $\theta_{y}=\pi$. The thermal state spectra on top are shifted vertically for reference. The electron spin spectra are centered around the detuning frequency $3 \mathrm{MHz}$. (b) ${ }^{13} \mathrm{C}$ spin spectra obtained by the pulse sequence shown in Fig. 3(b) without and with $U_{C N O T}$. The peaks appear at $\nu_{-}=0.11 \mathrm{MHz}$.

gives the frequency domain signal. Thus, as seen in the electron spin spectra in Fig. 4(a), the change of nuclear spin state resulted in a different frequency of the ESR lines in the case of $U_{C N O T}$ as compared to NOOP.

Since $U_{C N O T}$ targets the ${ }^{13} \mathrm{C}$, we also observed its effects on the ${ }^{13} \mathrm{C}$ by measuring the nuclear spin spectra using the pulse sequence in Fig. 3(b). The initial $180_{y}$ operation transforms $|0 \uparrow\rangle$ to $|-1 \uparrow\rangle \approx|-1\rangle \otimes\left(\left|\varphi_{-}\right\rangle-\left|\psi_{-}\right\rangle\right) / \sqrt{2}$. After implementing $U_{C N O T}$, we allowed the ${ }^{13} \mathrm{C}$ coherence between states $\left|\varphi_{-}\right\rangle$and $\left|\psi_{-}\right\rangle$to evolve for a variable time $t$, as shown in Fig. 3(c), and then applied another $180_{y}$ operation to the electron to bring the evolved state from $m_{S}=-1$ to $m_{S}=0$. The subsequent clean-up operation removed the population of $|0 \downarrow\rangle$ and allowed us to measure the remaining population of $|0 \uparrow\rangle$ with the readout laser pulse. The experimental ${ }^{13} \mathrm{C}$ spectra without and with $U_{C N O T}$ are shown in Fig. 4(b). The resonance frequency of the peak at $0.11 \mathrm{MHz}$ agree with the expected resonance frequency $\nu_{-}$of the ${ }^{13} \mathrm{C}$ for $m_{S}=-1$. Comparing with NOOP, the inverted amplitude shows that $U_{C N O T}$ flipped the ${ }^{13} \mathrm{C}$ states in $m_{S}=-1$. In Figs. $4(\mathrm{a}, \mathrm{b})$, we show the matching simulations, calculated for ideal pulses, scaled by a factor 0.8 .

As an additional test of the sequence for different input states, we first applied a selective rotation, when $m_{N}=1$ [39], of $\psi_{0}$ by an angle $\theta_{y}$ to generate the superposition state $\psi_{\theta}=\cos (\theta / 2)|0 \uparrow\rangle+\sin (\theta / 2)|-1 \uparrow\rangle$. As shown in Fig. 3(a), we then applied either a NOOP or $U_{C N O T}$. The latter transforms $\psi_{\theta}$ to $\cos (\theta / 2)|0 \uparrow\rangle-i \sin (\theta / 2) \mid-1 \downarrow$ \rangle , which is entangled for $\theta \neq n \pi$ with integer $n$. Ideally, the amplitude of the resonance line for the transition $|0 \downarrow\rangle$ $\leftrightarrow|1 \downarrow\rangle[40]$ is proportional to the population $P_{0 \downarrow}$. We thus determined $P_{0 \downarrow}$ and the results, which are shown in Fig. 5, demonstrate the effect of $U_{C N O T}$ for the 2 cases where the control qubit is $|0\rangle$ or $|-1\rangle$. Figure 5(a) shows $P_{0 \downarrow}$ after applying NOOP or $U_{C N O T}$ to $\psi_{\theta}$, as a function of $\theta$ in the absence of the $180_{y}$ operation indicated by the dotted box in Fig. 3(a). This pulse sequence allows us to measure the effect of $U_{C N O T}$ when the electron spin is $|0\rangle$. The curves for both cases are similar since $U_{C N O T}$ does not change the ${ }^{13} \mathrm{C}$ state when the electron spin is 


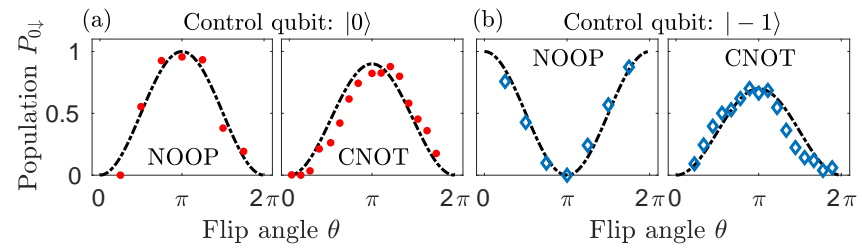

FIG. 5: $P_{0 \downarrow}$ as a function of $\theta$ corresponding to the pulse sequences shown in Fig. 3(a). The diamonds and solid circles are the experimental data, and the dashed lines are the matching simulations.

$|0\rangle$. In Fig. 5(b) we show the effect of $U_{C N O T}$ when the electron spin is $|-1\rangle$. To read out the population of $|-1 \downarrow\rangle$, we first applied a $180_{y}$ operation, as shown in Fig. 3(a) and then measured the electron spin FID in $m_{S}=\{0,1\}$. In this case, the $P_{0 \downarrow}$ vs $\theta$ curve flipped for $U_{C N O T}$ compared to NOOP, indicating the change of the ${ }^{13} \mathrm{C}$ state when the electron is in $|-1\rangle$. By fitting the experimental $P_{0 \downarrow}$ with the corresponding theoretical populations for various $\theta$ as shown in Fig. 5, we estimated the experimental infidelity due to $U_{C N O T}$ as $20 \%$ [32]. Discussion. - Our experiments convincingly show that the IC scheme is a very effective approach to implement operations in systems consisting of 3 types of qubits. The advantages of this approach will become even more important as the number of qubits increases. While a full implementation of the approach in large quantum registers is beyond the scope of this paper, we have tested the basic scheme through numerical simulations of gates in multiqubit systems with up to six qubits. The simulations show that the procedure scales relatively favorably with the size of the system [32]. For the 6-qubit system our method to control individual ${ }^{13} \mathrm{C}$ spins was efficient as it required 3-4 MW pulses and the total duration was $<30 \mu \mathrm{s}$. The theory $[25,41]$ regarding the bounds for the control overhead and the condition to retain efficiency for larger spin systems is explained in [32].

Conclusion. - We experimentally demonstrated full coherent control i.e, state initialization, gate implementa- tion and detection of the electron-nuclear spin system in the NV center of diamond using the methods of IC. We specifically chose a center with a small hyperfine coupling, some three orders of magnitude weaker than that of the nearest neighbor ${ }^{13} \mathrm{C}$ spins. The distance between the electron and ${ }^{13} \mathrm{C}$ is $\approx 0.89 \mathrm{~nm}$ [32]. These remote spins are much more abundant than the nearest neighbors and their relaxation times much longer. However, since their coupling to RF fields is also much weaker, direct RF excitation does not lead to efficient control operations. The IC techniques that we have demonstrated allow much faster controls and therefore overall higher fidelity - an essential prerequisite for scalable quantum systems. Specifically, we have implemented a Hadamard gate on ${ }^{13} \mathrm{C}$ and a CNOT gate, where the electron is the control qubit and ${ }^{13} \mathrm{C}$ the target qubit, using only a small number of MW pulses and delays. The above gate operations targeted the subspace $m_{S}=\{0,-1\}$ and $m_{N}=1$. If we consider the control state of the ${ }^{14} \mathrm{~N}$, i.e $m_{N}=1$, in the whole space with $m_{N}=\{0,-1,1\}$, then our $U_{C N O T}$ is a Toffolli gate in 12 dimensions. Since the total duration of the pulse sequence was well within the electron coherence time $\left(T_{2}^{*} \approx 20 \mu \mathrm{s}\right)$, additional coherence preserving control operations were not required. However, for complex algorithms consisting of many gates, it may be necessary to include DD. While we have implemented this scheme in the diamond NV center at room temperature in a small external magnetic field, it remains applicable over a much wider parameter range and can clearly be adapted to other quantum systems, thus opening the ways for many different implementations of advanced quantum algorithms using indirect control schemes.

This work was supported by the DFG through grants SU 192/34-1 and SU 192/31-1 and by the European Union's Horizon 2020 research and innovation programme under grant agreement No 828946. The publication reflects the opinion of the authors; the agency and the commission may not be held responsible for the information contained in it. SH thanks Dr T S Mahesh for fruitful discussions on genetic algorithms.
[1] M. A. Nielsen and I. Chuang, Quantum computation and quantum information (Cambridge University Press, Cambridge, England, 2002).

[2] J. Stolze and D. Suter, Quantum computing: a short course from theory to experiment (John Wiley \& Sons, Berlin, 2008).

[3] T. Gaebel, M. Domhan, I. Popa, C. Wittmann, P. Neumann, F. Jelezko, J. R. Rabeau, N. Stavrias, A. D. Greentree, S. Prawer, et al., Nature Physics 2, 408 (2006).

[4] P. Neumann, N. Mizuochi, F. Rempp, P. Hemmer, H. Watanabe, S. Yamasaki, V. Jacques, T. Gaebel, F. Jelezko, and J. Wrachtrup, science 320, 1326 (2008).

[5] J. Wrachtrup, S. Y. Kilin, and A. Nizovtsev, Optics and Spectroscopy 91, 429 (2001).
[6] D. Suter and F. Jelezko, Progress in nuclear magnetic resonance spectroscopy 98, 50 (2017).

[7] L. Childress, M. G. Dutt, J. Taylor, A. Zibrov, F. Jelezko, J. Wrachtrup, P. Hemmer, and M. Lukin, Science 314, 281 (2006).

[8] G. Fuchs, V. Dobrovitski, D. Toyli, F. Heremans, and D. Awschalom, Science 326, 1520 (2009).

[9] G. Balasubramanian, P. Neumann, D. Twitchen, M. Markham, R. Kolesov, N. Mizuochi, J. Isoya, J. Achard, J. Beck, J. Tissler, et al., Nature materials 8, 383 (2009).

[10] P. C. Maurer, G. Kucsko, C. Latta, L. Jiang, N. Y. Yao, S. D. Bennett, F. Pastawski, D. Hunger, N. Chisholm, M. Markham, et al., Science 336, 1283 (2012).

[11] E. Herbschleb, H. Kato, Y. Maruyama, T. Danjo, 
T. Makino, S. Yamasaki, I. Ohki, K. Hayashi, H. Morishita, M. Fujiwara, et al., Nature communications 10, 3766 (2019).

[12] C. Bradley, J. Randall, M. Abobeih, R. Berrevoets, M. Degen, M. Bakker, M. Markham, D. Twitchen, and T. Taminiau, Physical Review X 9, 031045 (2019).

[13] A. Gali, M. Fyta, and E. Kaxiras, Physical Review B 77, 155206 (2008).

[14] S. Meiboom and D. Gill, Review of scientific instruments 29, 688 (1958).

[15] G. S. Uhrig, Physical Review Letters 98, 100504 (2007).

[16] J. Zhang and D. Suter, Physical review letters 115, 110502 (2015).

[17] T. Van der Sar, Z. Wang, M. Blok, H. Bernien, T. Taminiau, D. Toyli, D. Lidar, D. Awschalom, R. Hanson, and V. Dobrovitski, Nature 484, 82 (2012).

[18] D. Suter and G. A. Álvarez, Rev. Mod. Phys. 88, 041001 (2016), URL http://link.aps.org/doi/10. 1103/RevModPhys . 88.041001.

[19] J. Zhang, A. M. Souza, F. D. Brandao, and D. Suter, Physical review letters 112, 050502 (2014).

[20] F. Jelezko, T. Gaebel, I. Popa, M. Domhan, A. Gruber, and J. Wrachtrup, Physical Review Letters 93, 130501 (2004).

[21] J. Shim, I. Niemeyer, J. Zhang, and D. Suter, Physical Review A 87, 012301 (2013).

[22] K. R. K. Rao and D. Suter, Physical Review B 94, 060101 (2016).

[23] J. Maze, J. Taylor, and M. Lukin, Physical Review B 78, 094303 (2008).

[24] P. Cappellaro, L. Jiang, J. Hodges, and M. D. Lukin, Physical review letters 102, 210502 (2009).

[25] N. Khaneja, Physical Review A 76, 032326 (2007).

[26] F. Wang, Y.-Y. Huang, Z.-Y. Zhang, C. Zu, P.-Y. Hou, X.-X. Yuan, W.-B. Wang, W.-G. Zhang, L. He, X.-Y. Chang, et al., Physical Review B 96, 134314 (2017).

[27] Y. Zhang, C. A. Ryan, R. Laflamme, and J. Baugh, Physical review letters 107, 170503 (2011).

[28] J. S. Hodges, J. C. Yang, C. Ramanathan, and D. G. Cory, Physical Review A 78, 010303 (2008).

[29] T. Taminiau, J. Wagenaar, T. Van der Sar, F. Jelezko, V. V. Dobrovitski, and R. Hanson, Physical review letters 109, 137602 (2012).

[30] C. D. Aiello and P. Cappellaro, Physical Review A 91, 042340 (2015).

[31] T. H. Taminiau, J. Cramer, T. van der Sar, V. V. Dobrovitski, and R. Hanson, Nature nanotechnology 9, 171
(2014).

[32] See the Supplemental Material for details of NV center system, Bloch Sphere representation of the gates, initial state determination, error estimation for CNOT, gates in multiqubit systems, spatial distance between the electron and the ${ }^{13} \mathrm{C}$, effects of operations on the ${ }^{14} \mathrm{~N}$ used in this work, which includes Refs. [38, 41-44].

[33] These operations are written in the computational basis states $\{|0 \uparrow\rangle,|0 \downarrow\rangle,|-1 \uparrow\rangle,|-1 \downarrow\rangle\}$ which is related to the energy eigenbasis by a transformation matrix $V=$ $|0\rangle\left\langle 0\left|\otimes E_{2}+\right|-1\right\rangle\langle-1| \otimes e^{-i \kappa_{-} I_{y}}$.

[34] M. Mitchell, An Introduction to Genetic Algorithms (MIT Press, Cambridge, MA, USA, 1998), ISBN 0262631857.

[35] J. Zhang, S. S. Hegde, and D. Suter, Physical Review Applied 12, 064047 (2019).

[36] J. Zhang, S. S. Hegde, and D. Suter, Physical Review A 98, $042302(2018)$.

[37] Here the hard $\theta_{y}$ operation refers to the operation in subspace $m_{S}=\{0,-1\}$ and $m_{N}=\{0,-1,1\}$. The corresponding Rabi frequency is $8 \mathrm{MHz}$.

[38] J. Cavanagh, W. J. Fairbrother, A. G. Palmer III, and N. J. Skelton, Protein NMR spectroscopy: principles and practice (Elsevier, 1995).

[39] If $\theta_{y}$ operation is a hard pulse as before, then for any value of $\theta$ not equal to integral multiple of $\pi$, this pulse creates electron spin coherence in $m_{N}=\{0,-1,1\}$ subspaces that evolve during the gate operations implemented in the system subspace. For simplicity, we here chose $\theta_{y}$ operation as selective pulse subjected to $m_{N}=1$ with Rabi frequency $0.5 \mathrm{MHz}$ as we vary $\theta$ value.

[40] Unlike the $4 \mathrm{ESR}$ peaks in $m_{S}=\{0,-1\}$ subspace, the ESR spectra in $m_{S}=\{0,1\}$ subspace has only two observable resonance peaks, where one peak corresponds to total population of state $|\uparrow\rangle$ and the other peak corresponds to total population of state $|\downarrow\rangle$ [see Ref. 32].

[41] F. Lowenthal, The Rocky Mountain Journal of Mathematics 1, 575 (1971).

[42] L. Jiang, J. M. Taylor, A. S. Sørensen, and M. D. Lukin, Physical Review A 76, 062323 (2007).

[43] J. Zhang, S. Saha, and D. Suter, Physical Review A 98, 052354 (2018).

[44] N. Mizuochi, P. Neumann, F. Rempp, J. Beck, V. Jacques, P. Siyushev, K. Nakamura, D. Twitchen, H. Watanabe, S. Yamasaki, et al., Physical review B 80, 041201 (2009) 